PROCEEDINGS OF THE AMERICAN MATHEMATICAL SOCIETY

Volume 124, Number 10, October 1996

\title{
SUR LES METRIQUES ADMETTANT LES PLANS COMME SURFACES MINIMALES
}

\author{
M. BEKKAR
}

(Communicated by Christopher Croke)

\begin{abstract}
We establish the system of partial differential equations satisfied by the riemannian metrics on open subsets of $\mathbb{R}^{3}$ which admit planes as minimal surfaces. This is a nonlinear system of 10 partial differential equations, with the euclidian metric as a particular solution. In a previous work, we solved this system for axially symmetrical metrics. In this paper we linearize the system at the euclidian metric and solve the linear system. We obtain a 20-dimensional space of solutions.
\end{abstract}

\section{INTRODUCTION}

La métrique euclidienne $d s^{2}=d x^{2}+d y^{2}+d z^{2}$ dans $\mathbb{R}^{3}$, notée $g_{E}$, admet tous les plans comme surfaces totalement géodésiques, donc minimales. Dans [B1] nous avons montré que les métriques $d s^{2}=d x^{2}+d y^{2}+(d z+k \omega)^{2}$ dans $\mathbb{R}^{3}$, où $\omega=$ $y d x-x d y$, admettent aussi tous les plans comme surfaces minimales (qui ne sont cependant plus totalement géodésiques). Ces métriques, notées $g_{H}^{k}$, s'obtiennent comme des métriques invariantes à gauche sur le groupe de Heisenberg. Robert Lutz a posé alors le problème de la détermination des métriques $g$ sur un ouvert $\Omega$ de $\mathbb{R}^{3}$ telles que toutes les traces $\mathcal{P} \cap \Omega$ des plans $\mathcal{P}$ de $\mathbb{R}^{3}$ sur $\Omega$ soient des surfaces minimales pour $g$. Notons par $\mathcal{M}$ l'espace de ces métriques.

Dans [B2], [B3] nous avons déterminé les éléments de $\mathcal{M}$ qui possèdent une symétrie axiale. On obtient une famille de métriques, notées $g_{\mu, \xi}$, dépendant de deux paramètres réels $\mu$ et $\xi$ et définies par :

$$
d s^{2}=\frac{1}{1-\left(\mu+\xi^{2}\right)\left(x^{2}+y^{2}\right)}\left(d x^{2}+d y^{2}-\mu \omega^{2}+2 \xi \omega d z+d z^{2}\right) .
$$

Lorsque $\mu+\xi^{2}>0$ la métrique $g_{\mu, \xi}$ est définie dans le cylindre $x^{2}+y^{2}<\left(\mu+\xi^{2}\right)^{-1}$. Lorsque $\mu+\xi^{2}=0$ on retrouve les métriques de Heisenberg $g_{H}^{\xi}$.

On établit dans cet article le système d'équations aux dérivées partielles, noté $\left(\mathcal{E}_{i j k}\right)$, que doivent satisfaire les éléments de $\mathcal{M}$. C'est un système non linéaire de 10 équations aux dérivées partielles, qui possède bien sûr comme solutions particulières la métrique euclidienne $g_{E}$ et les métriques à symétrie axiale $g_{\mu, \xi}$. On linéarise

Received by the editors March 15, 1995.

1991 Mathematics Subject Classification. Primary 49Q05, 53A10.

Key words and phrases. Minimal surfaces. 
ensuite ce système en sa solution $g_{E}$ et on montre que l'espace des solutions du linéarisé de $\left(\mathcal{E}_{i j k}\right)$ est de dimension $20{ }^{1}$

\section{Equations de l'espace $\mathcal{M}$}

Soit $d s^{2}=\sum g_{i j}\left(x_{1}, x_{2}, x_{3}\right) d x_{i} d x_{j}$ un élément de $\mathcal{M}$ où l'espace numérique $\mathbb{R}^{3}$ est rapporté à un système de coordonnées $\left(x_{1}, x_{2}, x_{3}\right)$. On note $m_{i j}$ le mineur du coefficient $g_{i j}$ dans la matrice $\mathbb{G}=\left(g_{i j}\right)$ des composantes du tenseur fondamental et $\Delta_{i j}=(-1)^{i+j} m_{i j}$ le complément algébrique de $g_{i j}$ dans $\mathbb{G}$. Notons $\mathbb{D}=\left(\Delta_{i j}\right)$ la matrice formée par les mineurs de $\mathbb{G}$ et $g:=\operatorname{det} \mathbb{G}, \Delta:=\operatorname{det} \mathbb{D}$. L'inverse de $\mathbb{G}$ s'écrit $\mathbb{G}^{-1}=\mathbb{D} / g$, d'où $g^{-1}=\Delta g^{-3}$ ce qui donne $\Delta=g^{2}$. On en déduit que $\mathbb{G}=g . \mathbb{D}^{-1}=\mathbb{M}\left(\Delta_{i j}\right) / \sqrt{\Delta}$, où $\mathbb{M}\left(\Delta_{i j}\right)$ désigne la matrice formée par les mineurs de $\mathbb{D}$. Ainsi les coefficients $g_{i j}$ s'obtiennent des $\Delta_{i j}$ par des formules du type $g_{11}=$ $\left(\Delta_{22} \Delta_{33}-\Delta_{23}^{2}\right) / \sqrt{\Delta}, g_{12}=\left(\Delta_{13} \Delta_{23}-\Delta_{12} \Delta_{33}\right) / \sqrt{\Delta}$, etc. La considération des coefficients $\Delta_{i j}$ comme inconnues de base s'impose naturellement dans les calculs qui nous font aboutir aux équations de l'espace $\mathcal{M}$. On a en effet le résultat suivant.

Théorème 1. Pour qu'une métrique riemannienne $d s^{2}=\sum g_{i j}\left(x_{1}, x_{2}, x_{3}\right) d x_{i} d x_{j}$ dans un ouvert $\Omega$ de $\mathbb{R}^{3}$ admette tous les plans comme surfaces minimales il faut et il suffit que les compléments algébriques $\Delta_{i j}$ des éléments $g_{i j}$ vérifient le système d'équations aux dérivées partielles suivant:

$$
\left(\mathcal{E}_{i j k}\right) \quad 2 \sum_{r=1}^{3} \Delta_{(i j} \Delta_{k) r, r}=\sum_{r=1}^{3} \Delta_{r(i} \Delta_{j k), r}
$$

où la virgule indique la dérivation partielle par rapport à la variable indiquée, et les parenthèses la symétrisation par rapport aux indices concernés.

Preuve. Soit $\left(g_{i j}\right)$ une métrique riemannienne sur un ouvert $\Omega$ de $\mathbb{R}^{3}$, qui admet les plans comme surfaces minimales. Soit $x_{3}=p x_{1}+q x_{2}+m+\varepsilon h\left(x_{1}, x_{2}\right)$, où $(p, q, m) \in$ $\mathbb{R}^{3}$ l'équation d'une surface $\mathcal{P}_{\varepsilon}$ proche du plan $\mathcal{P}$ d'équation $x_{3}=p x_{1}+q x_{2}+m$. On suppose que $\varepsilon$ est un paramètre qui varie dans un voisinage de 0 dans $\mathbb{R}$ et que $h$ est une fonction de classe $\mathcal{C}^{\infty}$ qui s'annule dans le complémentaire d'un domaine borné $\mathcal{D} \subset \mathbb{R}^{2}$ tel que les morceaux de surfaces $\mathcal{D}_{\varepsilon} \subset \mathcal{P}_{\varepsilon}$ et $\mathcal{D}_{0} \subset \mathcal{P}$ qui se projettent dans $\mathcal{D}$ par la projection $\left(x_{1}, x_{2}, x_{3}\right) \in \mathbb{R}^{3} \rightarrow\left(x_{1}, x_{2}\right) \in \mathbb{R}^{2}$ soient contenus dans $\Omega$. L'aire $\mathcal{A}(\varepsilon)$ du domaine $\mathcal{D}_{\varepsilon}$ est donnée par l'intégrale :

$$
\mathcal{A}(\varepsilon)=\iint_{\mathcal{D}} \sqrt{E_{\varepsilon} G_{\varepsilon}-F_{\varepsilon}^{2}} d x_{1} d x_{2}
$$

où

$$
\begin{aligned}
& E_{\varepsilon}=g_{11}+2 g_{13}\left(p+\varepsilon \frac{\partial h}{\partial x_{1}}\right)+g_{33}\left(p+\varepsilon \frac{\partial h}{\partial x_{1}}\right)^{2} \\
& F_{\varepsilon}=g_{12}+g_{23}\left(p+\varepsilon \frac{\partial h}{\partial x_{1}}\right)+g_{13}\left(q+\varepsilon \frac{\partial h}{\partial x_{2}}\right)+g_{33}\left(p+\varepsilon \frac{\partial h}{\partial x_{1}}\right)\left(q+\varepsilon \frac{\partial h}{\partial x_{2}}\right) \\
& G_{\varepsilon}=g_{22}+2 g_{23}\left(q+\varepsilon \frac{\partial h}{\partial x_{2}}\right)+g_{33}\left(q+\varepsilon \frac{\partial h}{\partial x_{2}}\right)^{2}
\end{aligned}
$$

\footnotetext{
${ }^{1}$ Dans [B2] un oubli de 3 constantes d'intégration m'a fait annoncer que la solution générale du champ linéarisé ne dépendait que de 17 paramètres. C'est R. E. Bryant qui m'a indiqué que la solution obtenue n'étant pas $S O(3)$-invariante, ne pouvait être la solution générale du système. Je le remercie pour m'avoir signalé cette erreur, pour ses remarques sur une première version de cet article et pour son intérêt au problème des métriques pour lesquelles les plans sont minimaux.
} 
La dérivée de la fonction $\mathcal{A}(\varepsilon)$ par rapport à $\varepsilon$, prise en $\varepsilon=0$, s'exprime à l'aide des compléments algébriques $\Delta_{i j}$ des $g_{i j}$. En effet on a :

$$
\left.\frac{\partial \mathcal{A}}{\partial \varepsilon}\right|_{\varepsilon=0}=\iint_{\mathcal{D}} \frac{1}{2 \sqrt{E_{0} G_{0}-F_{0}}}\left(\frac{\partial}{\partial \varepsilon}\left(E_{\varepsilon} G_{\varepsilon}-F_{\varepsilon}^{2}\right)\right)_{\left.\right|_{\varepsilon=0}} d x_{1} d x_{2}
$$

avec

$$
E_{0} G_{0}-F_{0}^{2}=\Delta_{33}-2 p \Delta_{13}-2 q \Delta_{23}+2 p q \Delta_{12}+p^{2} \Delta_{11}+q^{2} \Delta_{22}
$$

et

$$
\begin{gathered}
\frac{1}{2} \frac{\partial}{\partial \varepsilon}\left(E_{\varepsilon} G_{\varepsilon}-F_{\varepsilon}^{2}\right)_{\left.\right|_{\varepsilon=0}}=\left(-\Delta_{13}+q \Delta_{12}+p \Delta_{11}\right) \frac{\partial h}{\partial x_{1}}+\left(-\Delta_{23}+p \Delta_{12}+q \Delta_{22}\right) \frac{\partial h}{\partial x_{2}} \\
+\frac{1}{2} h \frac{\partial}{\partial x_{3}}\left(\Delta_{33}-2 p \Delta_{13}-2 q \Delta_{23}+2 p q \Delta_{12}+p^{2} \Delta_{11}+q^{2} \Delta_{22}\right) .
\end{gathered}
$$

Une intégration par parties, transforme cette dérivée en

$$
\frac{\partial \mathcal{A}}{\partial \varepsilon_{\left.\right|_{\varepsilon=0}}}=\iint_{\mathcal{D}} h\left(x_{1}, x_{2}\right) A\left(x_{1}, x_{2}\right) d x_{1} d x_{2}
$$

où $A\left(x_{1}, x_{2}\right)$ est la fonction :

$$
\begin{aligned}
A\left(x_{1}, x_{2}\right)= & \frac{\partial}{\partial x_{1}}\left(\frac{\Delta_{13}-q \Delta_{12}-p \Delta_{11}}{\sqrt{E_{0} G_{0}-F_{0}^{2}}}\right) \\
& +\frac{\partial}{\partial x_{2}}\left(\frac{\Delta_{23}-p \Delta_{12}-q \Delta_{22}}{\sqrt{E_{0} G_{0}-F_{0}^{2}}}\right)+\frac{1}{2 \sqrt{E_{0} G_{0}-F_{0}^{2}}} \frac{\partial}{\partial x_{3}}\left(E_{0} G_{0}-F_{0}^{2}\right) .
\end{aligned}
$$

Comme $h\left(x_{1}, x_{2}\right)$ est une fonction arbitraire, l'annulation de cette dérivée, qui exprime la propriété du plan $(\mathcal{P} \cap \Omega)$ d'être une surface minimale (voir $[\mathrm{O}]$ ), se traduit par l'équation $A\left(x_{1}, x_{2}\right)=0$, qui s'écrit aussi :

$$
\begin{gathered}
\left(\frac{\partial}{\partial x_{1}}\left(\Delta_{13}-q \Delta_{12}-p \Delta_{11}\right)+p \frac{\partial}{\partial x_{3}}\left(\Delta_{13}-q \Delta_{12}-p \Delta_{11}\right)\right. \\
+\frac{\partial}{\partial x_{2}}\left(\Delta_{23}-p \Delta_{12}-q \Delta_{22}\right)+q \frac{\partial}{\partial x_{3}}\left(\Delta_{23}-p \Delta_{12}-q \Delta_{22}\right) \\
\left.+\frac{1}{2} \frac{\partial}{\partial x_{3}}\left(\Delta_{33}-2 p \Delta_{13}-2 q \Delta_{23}+2 p q \Delta_{12}+p^{2} \Delta_{11}+q^{2} \Delta_{22}\right)\right)\left(E_{0} G_{0}-F_{0}^{2}\right) \\
-\frac{1}{2}\left(\frac{\partial}{\partial x_{1}}\left(E_{0} G_{0}-F_{0}^{2}\right)+p \frac{\partial}{\partial x_{3}}\left(E_{0} G_{0}-F_{0}^{2}\right)\right)\left(\Delta_{13}-q \Delta_{12}-p \Delta_{11}\right) \\
-\frac{1}{2}\left(\frac{\partial}{\partial x_{2}}\left(E_{0} G_{0}-F_{0}^{2}\right)+q \frac{\partial}{\partial x_{3}}\left(E_{0} G_{0}-F_{0}^{2}\right)\right)\left(\Delta_{23}-p \Delta_{12}-q \Delta_{22}\right)=0 .
\end{gathered}
$$

Ceci est un polynome de degré 4 en $p$ et $q$. Comme il doit être nul pour toutes les valeurs de $p$ et $q$, car tous les plans sont supposés minimaux, ses coefficients doivent être identiquement nuls. On obtient alors 10 équations. Trois parmi ces équations (lorsque les 3 indices sont égaux) sont de la forme :

$$
\Delta_{11}\left(\Delta_{11,1}+2 \Delta_{12,2}+2 \Delta_{13,3}\right)-\Delta_{12} \Delta_{11,2}-\Delta_{13} \Delta_{11,3}=0
$$


Six parmi ces équations (lorsque 2 des indices coïncident) sont de la forme :

$$
\Delta_{12}\left(4 \Delta_{23,3}+3 \Delta_{22,2}+2 \Delta_{12,1}\right)
$$

$$
+2 \Delta_{22}\left(\Delta_{11,1}+\Delta_{13,3}\right)-2 \Delta_{23} \Delta_{12,3}-\Delta_{13} \Delta_{22,3}-\Delta_{11} \Delta_{22,1}=0 .
$$

La dixième équation (lorsque les 3 indices sont différents) est :

$$
\begin{gathered}
\Delta_{12}\left(2 \Delta_{33,3}+\Delta_{13,1}+\Delta_{23,2}\right)+\Delta_{13}\left(2 \Delta_{22,2}+\Delta_{12,1}+\Delta_{23,3}\right) \\
+\Delta_{23}\left(2 \Delta_{11,1}+\Delta_{12,2}+\Delta_{13,3}\right)-\Delta_{11} \Delta_{23,1}-\Delta_{22} \Delta_{13,2}-\Delta_{33} \Delta_{12,3}=0 .
\end{gathered}
$$

On obtient ainsi le système $\left(\mathcal{E}_{i j k}\right)^{2}$

\section{Linearisation du Systeme $\left(\mathcal{E}_{i j k}\right)$}

Le linéarisé du système $\left(\mathcal{E}_{i j k}\right)$ en sa solution plate $g_{i j}=\delta_{i j}$ où $\delta_{i j}$ est le symbole de Kronecker, s'écrit, avec $\varepsilon_{i j}$ un tenseur symétrique représentant la première variation de la métrique $g_{i j}$ :

$$
\begin{gathered}
\varepsilon_{11,3}-2 \varepsilon_{13,1}+\varepsilon_{22,3}-2 \varepsilon_{23,2}=0, \\
\varepsilon_{33,1}-2 \varepsilon_{13,3}+\varepsilon_{22,1}-2 \varepsilon_{12,2}=0, \\
\varepsilon_{33,2}-2 \varepsilon_{23,3}+\varepsilon_{11,2}-2 \varepsilon_{12,1}=0, \\
\varepsilon_{11,1}-2 \varepsilon_{33,1}-\varepsilon_{22,1}+2 \varepsilon_{12,2}=0, \\
\varepsilon_{33,1}-2 \varepsilon_{13,3}-\varepsilon_{11,1}+2 \varepsilon_{22,1}=0, \\
\varepsilon_{33,3}-2 \varepsilon_{22,3}-\varepsilon_{11,3}+2 \varepsilon_{13,1}=0, \\
\varepsilon_{33,2}-2 \varepsilon_{23,3}-\varepsilon_{22,2}+2 \varepsilon_{11,2}=0, \\
\varepsilon_{22,2}-2 \varepsilon_{33,2}-\varepsilon_{11,2}+2 \varepsilon_{12,1}=0, \\
\varepsilon_{33,3}-2 \varepsilon_{11,3}-\varepsilon_{22,3}+2 \varepsilon_{23,2}=0, \\
\varepsilon_{13,2}+\varepsilon_{12,3}+\varepsilon_{23,1}=0 .
\end{gathered}
$$

Les solutions de ce système sont polynômiales de degré 2 et forment un espace vectoriel de dimension 20. Plus précisemment on a le résultat suivant.

Théorème 2. La solution générale du sytème $\mathcal{L}$ est donnée par :

$$
\begin{aligned}
\varepsilon_{11}= & A_{1}+\left(B_{2}+B_{3}\right) x_{1}+B_{1} x_{2}+C_{1} x_{3} \\
& +\left(D_{2} x_{3}+D_{3} x_{2}\right) x_{1}+\left(E_{2}+E_{3}\right) x_{1}^{2}+E_{1}\left(x_{2}^{2}+x_{3}^{2}\right), \\
\varepsilon_{22}= & A_{2}+\left(B_{1}+C_{3}\right) x_{2}+B_{2} x_{1}+C_{2} x_{3} \\
& +\left(D_{1} x_{3}+D_{3} x_{1}\right) x_{1}+\left(E_{1}+E_{3}\right) x_{2}^{2}+E_{2}\left(x_{1}^{2}+x_{3}^{2}\right),
\end{aligned}
$$

\footnotetext{
${ }^{2}$ Je dois à Th. Hangan cette écriture condensée du système. Je le remercie vivement pour son aide durant l'élaboration de ce travail.
} 


$$
\begin{gathered}
\varepsilon_{33}=A_{3}+\left(C_{1}+C_{2}\right) x_{3}+B_{3} x_{1}+C_{3} x_{2} \\
+\left(D_{1} x_{2}+D_{2} x_{1}\right) x_{1}+\left(E_{1}+E_{2}\right) x_{3}^{2}+E_{3}\left(x_{1}^{2}+x_{2}^{2}\right), \\
\varepsilon_{23}=F_{1}+G_{1} x_{1}+\frac{1}{2}\left[C_{1} x_{2}+B_{1} x_{3}+\left(D_{2} x_{2}+D_{3} x_{3}-D_{1} x_{1}\right) x_{1}\right]+E_{1} x_{2} x_{3}, \\
\varepsilon_{13}=F_{2}+G_{2} x_{2}+\frac{1}{2}\left[C_{2} x_{1}+B_{2} x_{3}+\left(D_{1} x_{1}+D_{3} x_{3}-D_{2} x_{2}\right) x_{2}\right]+E_{2} x_{1} x_{3}, \\
\varepsilon_{12}=F_{3}+G_{3} x_{3}+\frac{1}{2}\left[\left(C_{3} x_{1}+B_{3} x_{2}+\left(D_{1} x_{1}+D_{2} x_{2}-D_{3} x_{3}\right) x_{3}\right]+E_{3} x_{1} x_{2},\right.
\end{gathered}
$$

où les 21 constantes d'intégration $A_{i}, B_{i}, C_{i}, D_{i}, E_{i}, F_{i}$ et $G_{i}$ sont liées par la relation $G_{1}+G_{2}+G_{3}=0$.

Preuve. En considérant les combinaisons linéaires $(\mathcal{L} 2-\mathcal{L} 5+\mathcal{L} 4),(\mathcal{L} 3-\mathcal{L} 7+\mathcal{L} 8)$ et $(\mathcal{L} 1-\mathcal{L} 6+\mathcal{L} 9)$ on obtient les équations : $\left(\varepsilon_{11}-\varepsilon_{22}-\varepsilon_{33}\right)_{, 1}=0,\left(\varepsilon_{22}-\varepsilon_{33}-\varepsilon_{11}\right)_{, 2}=0$ et $\left(\varepsilon_{33}-\varepsilon_{11}-\varepsilon_{22}\right)_{, 3}=0$. Par conséquent $\varepsilon_{11}-\varepsilon_{22}-\varepsilon_{33}=-2 \alpha_{1}, \varepsilon_{22}-\varepsilon_{33}-\varepsilon_{11}=$ $-2 \alpha_{2}$ et $\varepsilon_{33}-\varepsilon_{11}-\varepsilon_{22}=-2 \alpha_{3}$, où $\alpha_{i}$ désigne une fonction intermédiaire, qui ne dépend que des variables $x_{j}$, avec $j \neq i$. On en déduit

$$
\varepsilon_{11}=\alpha_{2}+\alpha_{3}, \quad \varepsilon_{22}=\alpha_{1}+\alpha_{3}, \quad \varepsilon_{33}=\alpha_{1}+\alpha_{2} .
$$

Tenant compte de ces relations, les équations $(\mathcal{L} 4-\mathcal{L} 9)$ s'écrivent alors :

$$
\begin{aligned}
& 2 \varepsilon_{12,3}=\varepsilon_{33,1}=\alpha_{2,1}, \\
& 2 \varepsilon_{12,1}=\varepsilon_{33,2}=\alpha_{1,2}, \\
& 2 \varepsilon_{13,1}=\varepsilon_{22,3}=\alpha_{1,3}, \\
& 2 \varepsilon_{13,3}=\varepsilon_{22,1}=\alpha_{3,1}, \\
& 2 \varepsilon_{23,2}=\varepsilon_{11,3}=\alpha_{2,3}, \\
& 2 \varepsilon_{23,3}=\varepsilon_{11,2}=\alpha_{3,2} .
\end{aligned}
$$

En dérivant l'équation $\left(\mathcal{L}^{\prime} 8\right)$ par rapport à $x_{3}$ et l'équation $\left(\mathcal{L}^{\prime} 9\right)$ par rapport à $x_{2}$, on obtient la condition d'intégrabilité :

$$
\alpha_{2,33}=\alpha_{3,22} \text {. }
$$

En dérivant cette relation par rapport à $x_{3}$ on a $\alpha_{2,333}=0$, ce qui implique que $\alpha_{2}$ est un polynôme du second degré en $x_{3}$. En procédant de la même manière on montre que $\alpha_{2}$ est aussi un polynôme du second degré en $x_{1}$. De même $\alpha_{1}$ (resp. $\left.\alpha_{3}\right)$ est un polynôme de degré 2 en $\left(x_{2}, x_{3}\right)$ (resp. $\left(x_{1}, x_{2}\right)$ ). Par conséquent on a :

$$
\begin{aligned}
\alpha_{1}= & C_{0}^{1}+C_{2}^{1} x_{2}+C_{3}^{1} x_{3}+C_{22}^{1} x_{2}^{2} \\
& +C_{23}^{1} x_{2} x_{3}+C_{33}^{1} x_{3}^{2}+C_{223}^{1} x_{2}^{2} x_{3}+C_{233}^{1} x_{2} x_{3}^{2}+C_{2233}^{1} x_{2}^{2} x_{3}^{2}, \\
\alpha_{2}= & C_{0}^{2}+C_{1}^{2} x_{1}+C_{3}^{2} x_{3}+C_{11}^{2} x_{1}^{2} \\
& +C_{13}^{2} x_{1} x_{3}+C_{33}^{2} x_{3}^{2}+C_{113}^{2} x_{1}^{2} x_{3}+C_{133}^{2} x_{1} x_{3}^{2}+C_{1133}^{2} x_{1}^{2} x_{3}^{2}, \\
\alpha_{3}= & C_{0}^{3}+C_{1}^{3} x_{1}+C_{2}^{3} x_{2}+C_{11}^{3} x_{1}^{2} \\
& +C_{12}^{3} x_{1} x_{2}+C_{22}^{3} x_{2}^{2}+C_{112}^{3} x_{1}^{2} x_{2}+C_{122}^{3} x_{1} x_{2}^{2}+C_{1122}^{3} x_{1}^{2} x_{2}^{2} .
\end{aligned}
$$


En utilisant la relation d'intégrabilité (i) et les relations similaires $\alpha_{1,33}=\alpha_{3,11}$ et $\alpha_{2,11}=\alpha_{1,22}$, qui s'établissent de la même manière, on déduit, par identification des polynômes, les relations suivantes entre les coefficients :

$$
\begin{aligned}
& C_{11}^{3}=C_{33}^{1}, \quad C_{11}^{2}=C_{22}^{1}, \quad C_{33}^{2}=C_{22}^{3}, \\
& C_{112}^{3}=C_{233}^{1}, \quad C_{133}^{2}=C_{122}^{3}, \quad C_{113}^{2}=C_{233}^{1}, \\
& C_{2233}^{1}=C_{1133}^{2}=C_{1122}^{3} \text {. }
\end{aligned}
$$

En dérivant l'équation $(\mathcal{L} 10)$ par rapport à la variable $x_{3}$, on obtient

$$
\left(\varepsilon_{13,2}+\varepsilon_{12,3}+\varepsilon_{23,1}\right)_{, 3}=0
$$

qui donne, en utilisant les relations $\left(\mathcal{L}^{\prime} 7\right)$ et $\left(\mathcal{L}^{\prime} 9\right): \varepsilon_{12,33}=\alpha_{3,12}$. En dérivant cette relation par rapport à la variable $x_{3}$ on obtient $\varepsilon_{12,333}=0$, qui exprime que $\varepsilon_{12}$ est un polynôme de degré 2 en $x_{3}$. En dérivant maintenant l'équation $\left(\mathcal{L}^{\prime} 4\right)$ par rapport à $x_{2}$ et l'équation $\left(\mathcal{L}^{\prime} 5\right)$ par rapport à $x_{1}$ on obtient $\varepsilon_{12,22}=0$ et $\varepsilon_{12,11}=0$. Par conséquent $\varepsilon_{12}$ est un polynôme de degré 1 en $x_{1}$ et $x_{2}$ et on a

$$
\begin{aligned}
\varepsilon_{12}=K_{0}^{3}+K_{1}^{3} x_{1}+K_{2}^{3} x_{2}+K_{3}^{3} x_{3}+K_{13}^{3} x_{1} x_{3} & +K_{23}^{3} x_{2} x_{3}+K_{12}^{3} x_{1} x_{2} \\
& +K_{33}^{3} x_{3}^{2}+K_{133}^{3} x_{1} x_{3}^{2}+K_{233}^{3} x_{2} x_{3}^{2} .
\end{aligned}
$$

En utilisant les équations $\left(\mathcal{L}^{\prime} 4\right)$ et $\left(\mathcal{L}^{\prime} 5\right)$ on déduit, par identification des polynômes, les relations suivantes entre les coefficients :

$$
\begin{aligned}
& 2 K_{1}^{3}=C_{2}^{1}, \quad 2 K_{13}^{3}=C_{23}^{1}, \quad 2 K_{133}^{3}=C_{233}^{1}=0, \quad K_{12}^{3}=C_{22}^{1}, \\
& 2 K_{2}^{3}=C_{1}^{2}, \quad 2 K_{23}^{3}=C_{13}^{2}, \quad 2 K_{233}^{3}=C_{133}^{2}=0, \quad C_{2233}^{1}=0 .
\end{aligned}
$$

C'est ainsi que $\varepsilon_{12}$ s'écrit

$$
\varepsilon_{12}=K_{0}^{3}+K_{3}^{3} x_{3}+\frac{1}{2}\left(C_{2}^{1} x_{1}+C_{1}^{2} x_{2}+C_{23}^{1} x_{1} x_{3}+C_{13}^{2} x_{2} x_{3}\right)+C_{22}^{1} x_{1} x_{2}+K_{33}^{3} x_{3}^{2} .
$$

De la même manière on montre que :

$$
\begin{aligned}
\varepsilon_{13}= & K_{0}^{2}+K_{2}^{2} x_{2} \\
& +\frac{1}{2}\left(C_{3}^{1} x_{1}+C_{1}^{3} x_{3}+C_{23}^{1} x_{1} x_{2}+C_{12}^{3} x_{2} x_{3}\right)+C_{11}^{3} x_{1} x_{3}+K_{22}^{2} x_{2}^{2}, \\
\varepsilon_{23}= & K_{0}^{1} 2+K_{1}^{1} x_{1} \\
& +\frac{1}{2}\left(C_{3}^{2} x_{2}+C_{2}^{3} x_{3}+C_{13}^{2} x_{1} x_{2}+C_{12}^{3} x_{1} x_{3}\right)+C_{33}^{2} x_{2} x_{3}+K_{11}^{1} x_{1}^{2} .
\end{aligned}
$$

En utilisant l'équation $(\mathcal{L} 10)$ on déduit, par identification des polynômes, les relations suivantes entre les coefficients :

(iv)

$$
K_{1}^{1}+K_{2}^{2}+K_{3}^{3}=0, \quad 2 K_{11}^{1}=-C_{23}^{1}, \quad 2 K_{22}^{2}=-C_{13}^{2}, \quad 2 K_{33}^{3}=-C_{12}^{3} .
$$

En conclusion, en utilisant les relations (ii), (iii) et (iv) entre les coefficients, on peut exprimer les fonctions $\varepsilon_{i j}$ à l'aide des 20 paramètres libres

$$
\begin{array}{rllll}
A_{1}=C_{0}^{2}+C_{0}^{3}, & B_{1}=C_{2}^{3}, & C_{1}=C_{3}^{2}, & D_{1}=C_{23}^{1}, & E_{1}=C_{33}^{2}, \\
A_{2}=C_{0}^{1}+C_{0}^{3}, & B_{2}=C_{1}^{3}, & C_{2}=C_{1}^{3}, & D_{2}=C_{13}^{2}, & E_{2}=C_{11}^{3}, \\
A_{3}=C_{0}^{1}+C_{0}^{2}, & B_{3}=C_{1}^{2}, & C_{3}=C_{2}^{1}, & D_{3}=C_{12}^{3}, & E_{3}=C_{22}^{1}, \\
F_{1}=K_{0}^{1}, & F_{2}=K_{0}^{2}, & F_{3}=K_{0}^{3}, & G_{1}=K_{1}^{1}, & G_{2}=K_{2}^{2} .
\end{array}
$$

On obtient alors les expressions des solutions annoncées dans le théorème 2. 


\section{Remarques}

3.1. Un calcul élémentaire montre que, dans la première variation du tenseur de courbure qui correspond au tenseur $\varepsilon_{i j}$ n'interviennent que les constantes $D_{i}$ et $E_{i}$. En effet si on note les composantes de ce tenseur par $r_{i j k l}$ on a :

$$
\begin{gathered}
r_{1212}=E_{1}-E_{2}+E_{3}, \quad r_{1313}=-E_{1}+E_{2}+E_{3}, \quad r_{2323}=E_{1}+E_{2}-E_{3}, \\
r_{1213}=-D_{1}, \quad r_{2123}=-D_{2}, \quad r_{1323}=-D_{3} .
\end{gathered}
$$

3.2. Les résultats obtenus ici permettent de conjecturer que l'espace $\mathcal{M}$ est de "dimension" 20 aussi. En fait R. E. Bryant [Br], inspiré par une première version de cet article, a montré, avec une approche totalement différente de la notre, basée sur la théorie du repère mobile et la théorie des systèmes différentiels extérieurs de Elie Cartan, que l'espace $\mathcal{M}$ est effectivement de "dimension" 20, sans réussir toutefois à résoudre explicitement le système. Les résultats présentés dans cet article, basés sur une approche élémentaire et accessible à tous, peuvent être considérés donc comme une introduction et une motivation des résultats présentés dans [Br].

\section{REFERENCES}

[B1] M. Bekkar, Exemples de surfaces minimales dans l'espace de Heisenberg, Rend. Del. Sem. Univ. Cagliari 61-2 (1991), 123-130. MR 94b:53017

[B2] _ Métriques riemanniennes qui admettent le plan comme surface minimale, Thèse de Doctorat, Université de Haute Alsace, Mulhouse, Septembre 1993.

[B3] - Sur une caractérisation des métriques de Heisenberg, Comptes Rendus Acad. Sci. Paris, Série I 318 (1994), 1017-1019. MR 95f:53089

[Br] R. L. Bryant, On metrics in 3-space for which the planes are minimal, Preprint, Duke University, September 1994.

[O] R. Osserman, A survey of minimal surfaces, 1st ed., Van Nostrand, New York, 1969;. MR 41:934; 2nd ed., Dover, New York, 1986. MR 87j:53012

Université de Haute Alsace, 4 rue des Frères Lumière, F 68093 Mulhouse cedex, France et Université d’Oran Es-Sénia, Institut de Mathématiques, Oran, Algérie

E-mail address: M.Bekkar@univ-mulhouse.fr 